\title{
Particle-in-cell Monte Carlo simulations of an extreme ultraviolet radiation driven plasma
}

\section{Citation for published version (APA):}

Velden, van der, M. H. L., Brok, W. J. M., Mullen, van der, J. J. A. M., Goedheer, W. J., \& Banine, V. Y. (2006). Particle-in-cell Monte Carlo simulations of an extreme ultraviolet radiation driven plasma. Physical Review E Statistical, Nonlinear, and Soft Matter Physics, 73(3), 036406-1/6. [036406]. https://doi.org/10.1103/PhysRevE.73.036406

DOI:

10.1103/PhysRevE.73.036406

Document status and date:

Published: 01/01/2006

\section{Document Version:}

Publisher's PDF, also known as Version of Record (includes final page, issue and volume numbers)

\section{Please check the document version of this publication:}

- A submitted manuscript is the version of the article upon submission and before peer-review. There can be important differences between the submitted version and the official published version of record. People interested in the research are advised to contact the author for the final version of the publication, or visit the $\mathrm{DOI}$ to the publisher's website.

- The final author version and the galley proof are versions of the publication after peer review.

- The final published version features the final layout of the paper including the volume, issue and page numbers.

Link to publication

\section{General rights}

Copyright and moral rights for the publications made accessible in the public portal are retained by the authors and/or other copyright owners and it is a condition of accessing publications that users recognise and abide by the legal requirements associated with these rights.

- Users may download and print one copy of any publication from the public portal for the purpose of private study or research.

- You may not further distribute the material or use it for any profit-making activity or commercial gain

- You may freely distribute the URL identifying the publication in the public portal.

If the publication is distributed under the terms of Article 25fa of the Dutch Copyright Act, indicated by the "Taverne" license above, please follow below link for the End User Agreement:

www.tue.nl/taverne

Take down policy

If you believe that this document breaches copyright please contact us at:

openaccess@tue.nl

providing details and we will investigate your claim. 


\title{
Particle-in-cell Monte Carlo simulations of an extreme ultraviolet radiation driven plasma
}

\author{
M. H. L. van der Velden,* W. J. M. Brok, and J. J. A. M. van der Mullen \\ Department of Applied Physics, Eindhoven University of Technology, P.O. Box 513, 5600 MB Eindhoven, The Netherlands \\ W. J. Goedheer \\ FOM-Institute for Plasma Physics Rijnhuizen, P.O. Box 1207, 3430 BE Nieuwegein, The Netherlands \\ V. Banine \\ ASML Netherlands B.V., De Run 6501, 5504 DR Veldhoven, The Netherlands
}

(Received 4 November 2005; published 14 March 2006)

\begin{abstract}
A self-consistent kinetic particle-in-cell model has been developed to describe a radiation driven plasma. Collisions between charged species and the neutral background are represented statistically by Monte Carlo collisions. The weakly ionized plasma is formed when extreme ultraviolet radiation coming from a pulsed discharge photoionizes a low pressure argon gas. The presence of a plasma close to optical components is potentially dangerous in case the ions that are accelerated in the plasma sheath gain enough energy to sputter the optics. The simulations predict the plasma parameters and notably the energy at which ions impact on the plasma boundaries. Finally, sputter rates are estimated on the basis of two sputtering models.
\end{abstract}

DOI: 10.1103/PhysRevE.73.036406

PACS number(s): 52.65.Rr, 52.65.Pp, 52.40.Kh

\section{INTRODUCTION}

To meet the semiconductor industries continuous demand for printing ever-smaller structures on silicon wafers, future lithographic tools are designed to operate using extreme ultraviolet (EUV) radiation in a $2 \%$ bandwidth around the central wavelength of $13.5 \mathrm{~nm}$. Since no material is sufficiently transparent to EUV radiation, refractive optics cannot be used and instead multilayer coated mirrors are required [1].

Additionally, as the mean free path of EUV radiation at atmospheric pressures is very short, the whole optical path is contained in a vacuum system. The residual argon background gas at a pressure of 0.1 to $1 \mathrm{~Pa}$, is (partially) photoionized by the EUV radiation, creating a weakly-ionized plasma. The EUV radiation is generated by a hollow cathode discharge [2,3], which generates EUV pulses of approximately 100 ns duration at a repetition rate of typically $1 \mathrm{kHz}$. Consequently, the plasma will show strong time dependence.

As a result of the low plasma density $\left(n_{e} \approx 10^{15} \mathrm{~m}^{-3}\right)$ recombination will occur predominantly on the walls of the tool. Like in any bounded plasma, a plasma sheath will develop in which the ions will be accelerated towards the vessel walls. The plasma is potentially dangerous to the optical elements in case the ions gain enough energy to damage the multilayer mirrors through physical sputtering. A plasma model is applied to calculate the flux and energy of the ions impacting on the mirror.

The low plasma density and the strong time dependence imply that the plasma is far from equilibrium. Furthermore, at such a low pressure the plasma is in the nonlocal regime and a kinetic model is needed for an accurate description. Particle-in-cell (PIC) kinetic models provide a way to selfconsistently calculate the fields and the energy and velocity distributions, without the need for equilibrium assumptions

\footnotetext{
*Electronic address: M.H.L.v.d.Velden@tue.n1
}

$[4,5]$. Collisions can be represented statistically by combining PIC methods with Monte Carlo collisions (MCC) [6].

\section{DESCRIPTION OF THE MODEL}

The plasma is simulated using a PIC Monte Carlo code, which is one dimensional in configuration and three dimensional in velocity space. Electrons and argon ions are computationally represented by "superparticles." Each superparticle corresponds to typically $10^{9}$ real particles. The computational grid divides the plasma into a number of cells. Each cell must contain at least 100 particles to ensure proper statistics.

The PIC scheme will be described only briefly as this is well documented elsewhere [4,5]. The general scheme of the explicit PIC model is shown in Fig. 1. For each time step $\Delta t$ we have the following:

(i) Charges are assigned to the nodes of the computational grid by a linear weighing, the so-called cloud-in-cell scheme [7].

(ii) The Poisson equation is solved to obtain the electric field at the nodes.

(iii) To determine the force on each particle a linear weighing is applied to find the electric field at each particle position.

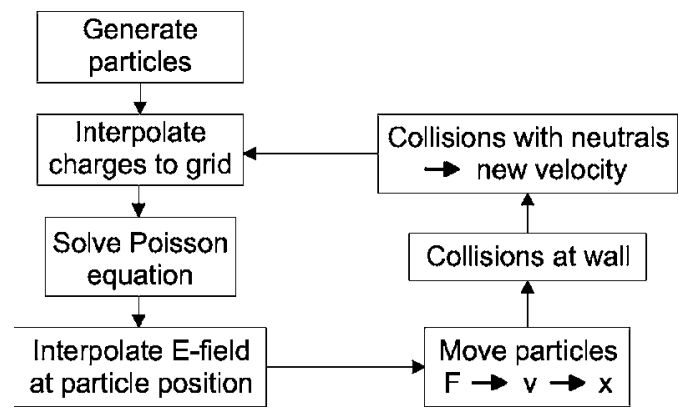

FIG. 1. Particle-in-cell scheme. 
(iv) The Newton equations of motions are applied to advance the position and velocity of the particles. Positions and fields are defined at integral time levels whereas velocities are defined at half-integral time levels.

(v) Particles that have moved beyond the boundaries of the computational grid are removed.

(vi) The MCC routine checks if a particle has collided and adjusts the velocity accordingly.

\section{A. Monte Carlo collisions}

In the model, charged particles collide with neutrals only. Because of the low plasma density, collisions between charged particles can be neglected. A collision is treated here as an instantaneous process that only changes the particle's velocity. Assume that the particle species have $N$ types of collisions with the background gas. The total collision cross section $\sigma_{T}(E)$ is the sum

$$
\sigma_{T}(E)=\sum_{i=1}^{N} \sigma_{i}(E),
$$

where $E$ is the kinetic energy of the incident particle and $\sigma_{i}(E)$ the cross-section for the $i$ th type of collision. The flight time between collisions depends on the background density $n(x)$ and the energy and is equal to the inverse of the collision frequency $\nu$

$$
\nu(x, E)=n(x) \sigma_{T}(E) \sqrt{\frac{2 E}{m}},
$$

with $m$ the incident particle mass. The probability $P(t) d t$ that a particle will experience a collision within an infinitesimal time interval $d t$ after having traveled through the background gas for a time $t$ is

$$
P(t) d t=\nu \exp (-\nu t) d t .
$$

To determine when a collision takes place we have to integrate Eq. (3) along the particle trajectory. Numerically this is very time consuming because the background density and the kinetic energy of the particle change along its path and we have to calculate $\nu$ after each time step $\Delta t$ of the PIC loop. Therefore, we use a numerical trick called "null collision method" [6] to speed up the calculations. Hereto, an extra collision type is introduced, which has a cross section such that, when added to Eq. (1), results in a total collision frequency with a constant value $\nu_{m}$

$$
\nu_{m}=\max _{x, E}\left\{n(x) \sigma_{T}(E) \sqrt{\frac{2 E}{m}}\right\} .
$$

In our case the plasma is only weakly ionized with a homogeneous background density and we only have to obtain the maximum over $E$. The extra collision type is called "null collision" as no real interaction occurs. To obtain the time $t_{m}$ to the next collision we integrate the right-hand side of Eq. (3) from zero to $t_{m}$ replacing $\nu$ by $\nu_{m}$ and equate the left-hand side to $R \in[0,1)$, a random number between zero and one. All random numbers mentioned hereafter, are drawn from this interval. Solving for $t_{m}$ gives

$$
t_{m}=-\frac{\ln (1-R)}{\nu_{m}} .
$$

Upon creation at time $t_{0}$, each individual particle is assigned a collision time $t_{c}=t_{0}+t_{m}$. After each time step of the PIC loop it is evaluated whether the particle needs to collide, i.e., it is checked if $t \geqslant t_{c}$. If so, the collision frequency $\nu_{i}(E)$ of each process at $t_{c}$ is calculated to determine the collision type from

$$
\begin{gathered}
0<R \leqslant \nu_{1}(E) / \nu_{m} \quad \text { type } 1 \\
\nu_{1}(E) / \nu_{m}<R \leqslant \sum_{i=1}^{2} \nu_{i}(E) / \nu_{m} \quad \text { type } 2, \\
\vdots
\end{gathered}
$$

$$
\sum_{i=1}^{N} \nu_{i}(E) / \nu_{m}<R \quad \text { null collision }
$$

with $R$ a random number. Once the collision type is determined the velocity of the particle is adjusted accordingly. After the collision the time to the next collision $t_{m}$ [using Eq. (5)] is added to the particle's collision time.

\section{B. Collision types}

The following binary collision types are taken into account.

(i) Elastic electron-neutral collisions: $e^{-}+\mathrm{Ar} \rightarrow e^{-}+\mathrm{Ar}$.

(ii) Inelastic electron-neutral collisions: $e^{-}+\mathrm{Ar} \rightarrow e^{-}+\mathrm{Ar}^{*}$.

(iii) Electron-impact ionization collisions: $e^{-}+\mathrm{Ar} \rightarrow 2 e^{-}$ $+\mathrm{Ar}^{+}$.

(iv) Elastic ion neutral collisions: $\mathrm{Ar}^{+}+\mathrm{Ar} \rightarrow \mathrm{Ar}^{+}+\mathrm{Ar}$.

(v) Charge-exchange collisions: $\mathrm{Ar}^{+}+\mathrm{Ar} \rightarrow \mathrm{Ar}+\mathrm{Ar}^{+}$.

Cross sections for these processes are the same as the ones used by Phelps [8] for their description of cold-cathode discharges over a wide range of electric field to gas density ratios $(E / n=15 \mathrm{Td}$ to $100 \mathrm{kTd})$. The collision frequencies for electron-argon scattering associated with these cross sections are plotted in Fig. 2.

\section{Electron-neutral collisions}

\section{Elastic collisions}

In the low energy limit, electron-neutral scattering is isotropic, but at higher energies the scatter angle $\theta$ will increasingly be in the forward direction. In the first Bornapproximation [9] the quantum mechanical differential cross section $\sigma(\varepsilon, \theta)$ for screened Coulomb electron-neutral scattering is given by $[10]$

$$
\frac{\sigma(\varepsilon, \theta)}{\sigma(\varepsilon)}=\frac{1}{4 \pi} \frac{1+8 \varepsilon}{(1+4 \varepsilon-4 \varepsilon \cos \theta)^{2}},
$$

with $\varepsilon=E / E_{0}$ a dimensionless energy parameter and $E_{0}=27.21 \mathrm{eV}$ the atomic unit of energy, which can be expressed in fundamental constants. For atomic collisions, scat- 


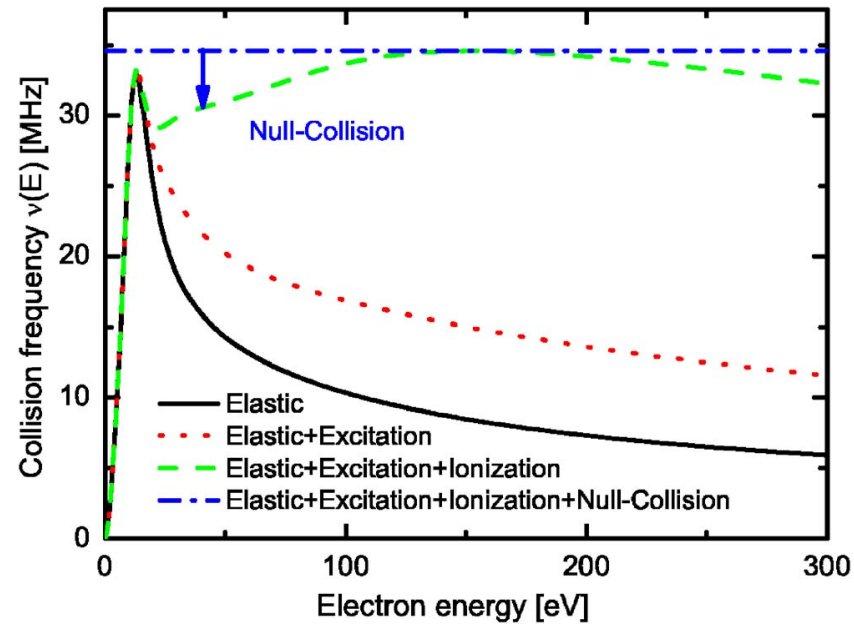

FIG. 2. (Color online) Collision frequencies for electron-argon scattering based on the cross sections of Phelps [8]. Solid line: elastic scattering. Dotted line: sum of elastic and inelastic scattering. Dashed line: sum of elastic, inelastic, and ionization processes. Dashed-dotted line: maximum collision cross section $\nu_{m}$.

tering is isotropic in the azimuthal direction and therefore the differential cross section does not depend on the $\varphi$ coordinate. The scattering angle $\varphi$ in the azimuthal direction is found from $\varphi=2 \pi R$ with $R$ a random number. To find the scattering angle $\theta$ of an electron in the polar direction we sample the angle from the differential cross section. Hereto, we first calculate the cumulative probability by integration of Eq. (7) over all solid angles with $\theta^{\prime} \in[0, \theta]$ and equate the result to a random number

$$
R=2 \pi \int_{0}^{\theta} \frac{\sigma\left(\varepsilon, \theta^{\prime}\right)}{\sigma(\varepsilon)} \sin \left(\theta^{\prime}\right) d \theta^{\prime} .
$$

Solving for $\theta$ yields

$$
\theta=\operatorname{arcos}\left(1-\frac{2 R}{1+8 \varepsilon(1-R)}\right) .
$$

This expression gives the scattering angle for an electron with dimensionless energy $\varepsilon$ in the center-of-mass frame. Because the electron-neutral mass ratio is a very small number, energy transfer from the electron to the neutral will be small and the scattering angle in the laboratory frame will be approximately the same.

\section{Excitation}

In inelastic collisions, energy is transferred to the internal states of the argon atom. In the model all internal states are merged into one single excitation level with an energy of $E_{\text {exc }}=11.5 \mathrm{eV}$ above the argon ground state [8]. First, the excitation energy is subtracted from the electron kinetic energy after which the scattering angle is determined from Eq. (9).

\section{Ionization}

If the kinetic energy $E_{\text {in }}$ of the incoming electron is above the argon ionization energy $E_{\text {ion }}$ an ionizing electron-argon collision can occur, in which an electron-ion pair is created. The excess energy $E_{i n}-E_{i o n}$ is carried away by the two electrons, whereas the kinetic energy of the ion will be equal to the initial (thermal) energy of the neutral. In the model, the velocity of the target neutral is sampled from a Maxwellian velocity distribution that corresponds to a temperature of $T=300 \mathrm{~K}$.

It is not possible to predict how the excess energy will be distributed between the incoming and ejected electron. Therefore, we apply an empirical formula for the distribution function based on the work of Opal [11] as mentioned in Surendra and Graves [12]

$$
S\left(E_{i n}, E_{e j}\right)=\frac{A}{E_{e j}^{2}+B^{2}\left(E_{i n}\right)},
$$

with $A$ a normalization constant that can be obtained from integration over $E_{e j}$ from 0 to the maximum energy $E_{\text {max }}=\left(E_{\text {in }}-E_{\text {ion }}\right) / 2$, which is half the excess energy due to the indistinguishability of the two electrons involved

$$
\begin{aligned}
A & =\left\{\int_{0}^{E_{\max }} S\left(E_{i n}, E_{e j}\right) d E_{e j}\right\}^{-1}, \\
& =\frac{B\left(E_{i n}\right)}{\arctan \left[\left(E_{i n}-E_{i o n}\right) / 2 B\left(E_{i n}\right)\right]} .
\end{aligned}
$$

For argon $B\left(E_{\text {in }}\right) \approx 10 \mathrm{eV}$ in the energy range 1 to $70 \mathrm{eV}$ [11].

Upon collision, we first obtain the energy of the ejected electron by drawing a random number $R$ to sample the distribution of Eq. (10) according to

$$
E_{e j}=B\left(E_{i n}\right) \tan \left[R \arctan \left(E_{\text {max }} / B\left(E_{i n}\right)\right] .\right.
$$

The ejected electron is created at the position of the incoming electron and the scattering angle is determined by Eq. (9). Next, the energy of the incoming electron is reduced by the sum of the ionization energy and the energy of the ejected electron after which the scattering angle is again determined from Eq. (9).

\section{Ion-neutral collisions}

Because the masses of ions and neutrals are almost the same, the collisional energy exchange between them is considerable and has to be properly taken into account. Therefore, collisions between ions and argon atoms are treated in the center-of-mass frame. The velocities of the target neutrals are sampled from a Maxwellian velocity distribution at room temperature $(T=300 \mathrm{~K})$. For ion collisions we assume scattering to be isotropic with a scattering angle in the center-ofmass frame equal to

$$
\theta=\operatorname{arcos}(1-2 R),
$$

with $R$ a random number. After the collision the velocity of the argon ion is transferred back to the laboratory frame.

\section{Generation of particles}

Figure 3 shows a schematic of the geometry used in the simulations. The radiation driven plasma is bounded by a 


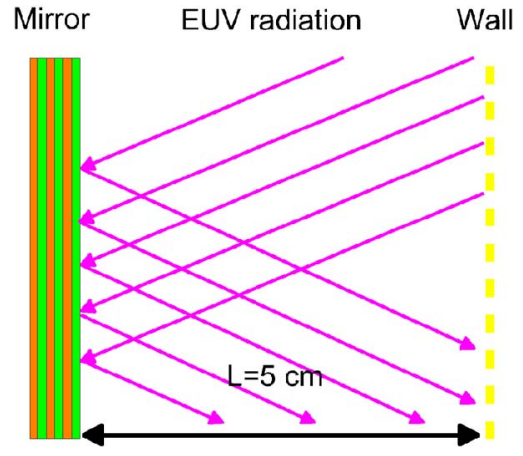

FIG. 3. (Color online) Schematic of the geometry used in simulations. The EUV radiation is partially reflected by the mirror. It is assumed that the EUV radiation between the mirror on the left and a plain wall on the right to be monochromatic and homogeneous in intensity.

multilayer mirror on the left side and a metal wall on the right side. The top layer of the mirror is assumed to be ruthenium, because in EUV lithography this material is often applied as a capping layer (thickness of $\approx 1.5 \mathrm{~nm}$ ) to provide a barrier against oxidation of the underlying Mo/Si multilayers [13]. Both the multilayer mirror and wall are assumed to be grounded. At the multilayer mirror the EUV radiation is partially reflected, with a reflection coefficient of typically $R_{m l}=68 \%$ [14]. The remaining $32 \%$ of the radiation is absorbed by the mirror material and is transformed mainly into heat.

The spatial distribution of the EUV radiation is assumed to be homogeneous, whereas the temporal distribution $G(t)$ is modeled with a cutoff Gaussian with a total duration of $2 \tau=100 \mathrm{~ns}$

$$
G(t)=\frac{\alpha I_{p}}{\tau} \exp \left[-\frac{(t-\tau)^{2}}{2 \tau^{2}}\right] \text { for } 0<t<2 \tau
$$

with $\alpha$ a numerical constant to normalize $G(t)$ to the pulseaveraged EUV intensity $I_{p}$.

Electron-ion pairs are generated as a result of photoionization of the argon background gas. The EUV photon energy of $h \nu=92 \mathrm{eV}$ is well above the argon ionization energy $E_{\text {ion }}=15.8 \mathrm{eV}$. Consequently, in the volume argon can be photoionized by EUV radiation, creating a fast electron with kinetic energy $T_{e}=h \nu-E_{i o n}$ and a slow $\mathrm{Ar}^{+}$ion, with kinetic energy equal to the thermal energy of argon at room temperature.

The total number $N_{p i}$ of photoionization events per EUV pulse per $m^{3}$ is equal to

$$
N_{p i}=\frac{I_{p}}{h \nu L}\left[1-\exp \left(-n_{A r} \sigma_{p h} L_{o p t}\right)\right],
$$

with $I_{p}$ the pulse averaged EUV intensity, $n_{A r}$ the argon density, $\sigma_{p h}$ the photoionization cross section [15], and $L_{\text {opt }}=L\left(1+R_{m l}\right)$ the effective optical path length that takes into account the partial reflection of the EUV beam from the mirror as indicated in Fig. 3.
TABLE I. Simulation parameters.

\begin{tabular}{lcc}
\hline \hline Background density & $n_{A r}$ & $1.2 \times 10^{20} \mathrm{~m}^{3}$ \\
EUV intensity & $I_{p}$ & $6 \times 10^{-4} \mathrm{~W} \mathrm{~m}^{-2}$ \\
Time step & $\Delta t$ & $1 \times 10^{-12} \mathrm{~s}$ \\
Particle weight & $P W$ & $10^{9}$ \\
Number of cells & $N_{c}$ & 300 \\
Length & $L$ & $5 \times 10^{-2} \mathrm{~m}$ \\
\hline \hline
\end{tabular}

\section{RESULTS/DISCUSSION}

The simulation parameters are listed in Table I. The chosen EUV intensity is typical for our laboratory setup. In our simulations we set the argon background pressure at $0.5 \mathrm{~Pa}$. At this low pressure the plasma will be in the nonlocal regime and the plasma sheath will be almost, but not quite, collisionless, as we estimate the average number of ionneutral collisions for an ion crossing the sheath with an energy between 20 and $100 \mathrm{eV}$ to be $\approx 0.2$.

We divide the length of the computational domain into $N_{c}=300$ cells, so that the cell size is smaller than the Debye screening length, which is $\lambda_{D} \approx 5 \times 10^{-4} \mathrm{~m}$ for our application. Each species is represented by $10^{5}$ superparticles to ensure proper statistics. The time step of the PIC-loop must be small compared to the time it takes a fast electron to travel across a cell, which for a $100 \mathrm{eV}$ electron and for our cell size corresponds to $\approx 30 \mathrm{ps}$. This is two orders of magnitude smaller than the fastest time scale of the plasma, which is given by the inverse of the plasma electron frequency.

Figure 4 shows the cell-averaged energy of the plasma electrons as a function of position at various times. Initially, the plasma is very hot and the energy of the electrons is close to $h \nu-E_{\text {ion }} \approx 76 \mathrm{eV}$. The plasma cools mainly due to inelastic and ionizing collisions of electrons with the neutral background. After the $100 \mathrm{~ns}$ long EUV pulse the photoionization process ceases and the average electron energy decreases rapidly as no more fast electrons are generated. As can be seen in Fig. 4, the spatial fluctuations of the average electron energy decrease with time as more superparticles are added

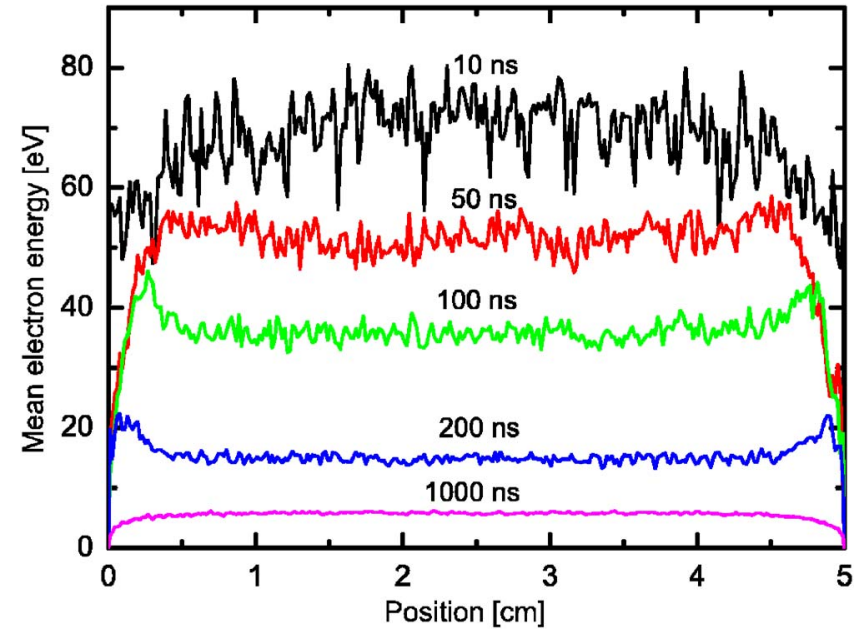

FIG. 4. (Color online) Cell-averaged electron energy profile at five different times. 


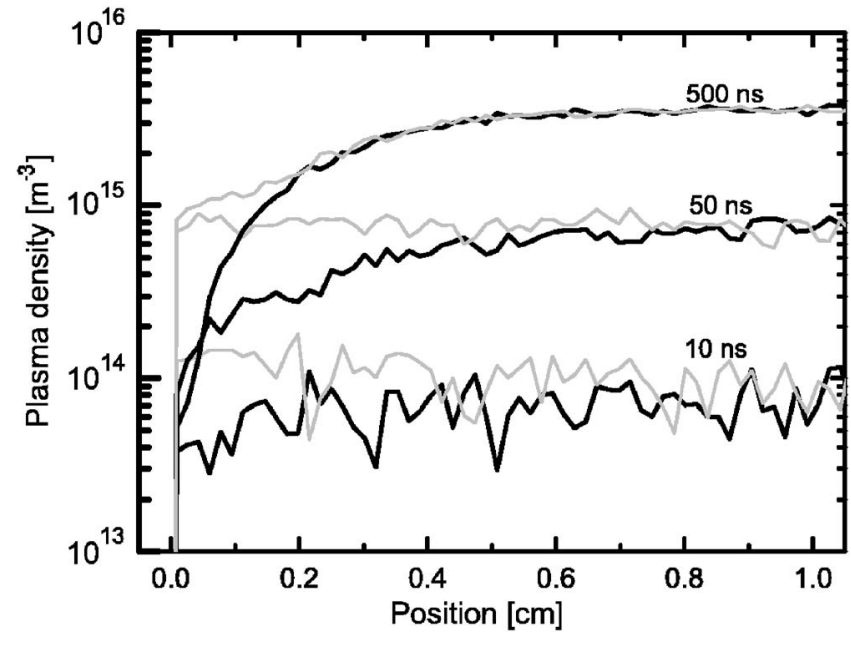

FIG. 5. Plasma density profile near the mirror at three different times. $t=10 \mathrm{~ns}$ (bottom), $t=50 \mathrm{~ns}$ (middle), and $t=500 \mathrm{~ns}$ (top). The thick lines represent electron density, whereas the thin lines indicate ion density.

to the simulation, thereby improving the statistical representation.

The development of the plasma sheath can be seen in Fig. 5 , which shows the plasma density profile near the mirror region at three different times. Near the mirror, the ion density exceeds the electron density and a positive space charge region forms as the highly mobile electrons have escaped to the wall, leaving the more inert ions behind. At the end of the EUV pulse, most electrons still have sufficient kinetic energy to cause further ionization processes. That is why the maximum plasma density $n_{e}=4 \times 10^{15} \mathrm{~m}^{-3}$ is reached long after the EUV pulse at $t=500 \mathrm{~ns}$. After that, the plasma density starts to decay on a time scale that is short compared to the time between EUV pulses.

The formation of the plasma sheath can also be studied by considering the potential, shown in Fig. 6. The maximum plasma potential of $\approx 80$ volts is reached during the EUV pulse. The plasma is at a positive potential with respect to the

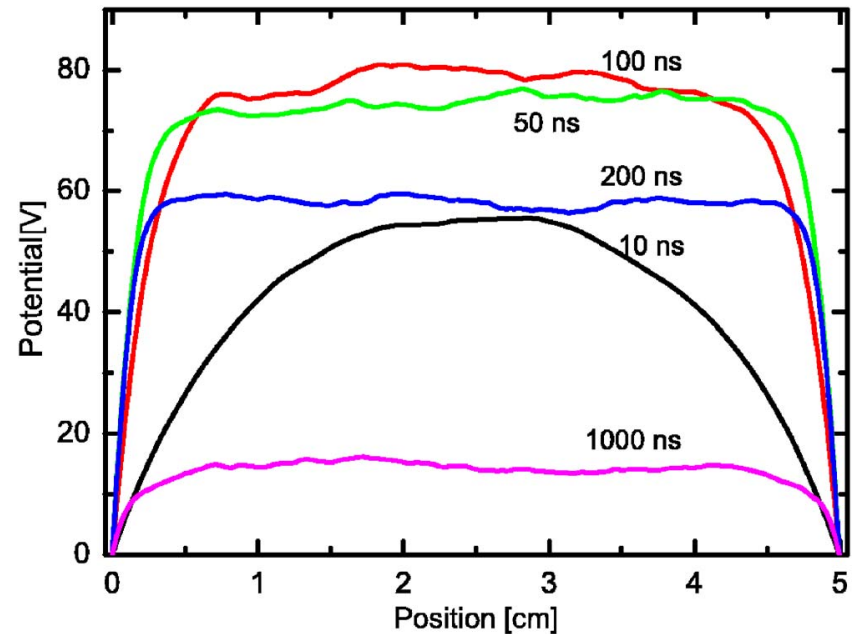

FIG. 6. (Color online) Potential profile at five different times. At $t=10 \mathrm{~ns}$ (thick solid line), $t=50 \mathrm{~ns}$ (dashed line), $t=100 \mathrm{~ns}$ (dotted line), $t=200 \mathrm{~ns}$ (dash-dotted line), and $t=1000 \mathrm{~ns}$ (thin solid line). walls as a result of the positive space charge in the sheath, thus creating an electric field in the sheath that directs electrons into the plasma and accelerates ions towards the walls. After the EUV pulse $(t>100 \mathrm{~ns})$ the plasma potential gradually decreases, because the charge separation in the plasma sheath is reduced as a result of the decrease in average electron energy.

In Fig. 7 the kinetic energy of ions impacting on the mirror is shown as a function of time. For clarity's sake, the moving average over 50 consecutive ion impacts is taken. The dotted line shows the temporal shape of the EUV pulse. Due to their inertia the ions reach the mirror after the EUVpulse. The ion impact energy reaches a maximum of $\approx 40 \mathrm{eV}$ at $t=270 \mathrm{~ns}$.

Whether this ion bombardment translates into mirror damage goes beyond this model. It needs to be determined experimentally. However, as a first order estimation we can apply the Bohdansky [16] model for light ion sputtering. This semiempirical model gives the sputter yield $Y(E)$, i.e., the number of atoms removed from the solid per incoming ion with energy $E$. Only ions with an energy above the sputter threshold contribute. The sputter threshold can be calculated from

$$
E_{t h r}= \begin{cases}\frac{U_{s}}{\lambda(1-\lambda)} & \text { for }\left(\frac{m_{1}}{m_{2}}\right)<0.3 \\ 8 U_{s}\left(\frac{m_{1}}{m_{2}}\right)^{2 / 5} & \text { for }\left(\frac{m_{1}}{m_{2}}\right)>0.3,\end{cases}
$$

with $U_{s}$ the surface binding energy of the solid which can be approximated by the sublimation heat and with $\lambda$ the fraction of energy that is transferred from the projectile (with mass $m_{1}$ ) to the target atom (with mass $m_{2}$ ) in case of a head-on collision

$$
\lambda=\frac{4 m_{1} m_{2}}{\left(m_{1}+m_{2}\right)^{2}} .
$$

For $\mathrm{Ar}^{+}$on $\mathrm{Ru} \lambda=0.812, U_{s}=6.74 \mathrm{eV}$ and $E_{t h r}=37.2 \mathrm{eV}$.

In Fig. 7 the sputter threshold is indicated by the bold dashed line. Only a fraction of the ions has an impact energy

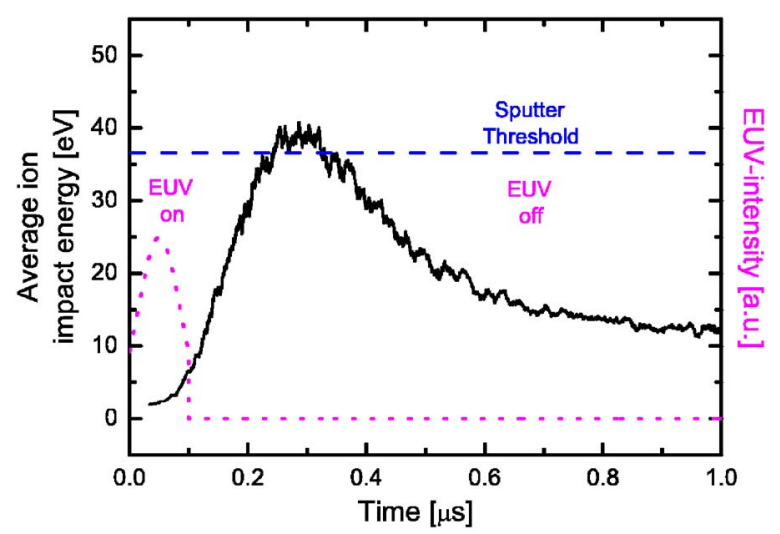

FIG. 7. (Color online) Solid line: the energy in eV's of ions that have impacted on the mirror. For clarity, the depicted energy is the moving average over fifty consecutive ion impacts. Dotted line: the temporal profile of the EUV intensity in arbitrary units. Dashed line: the Bohdansky sputter threshold. 


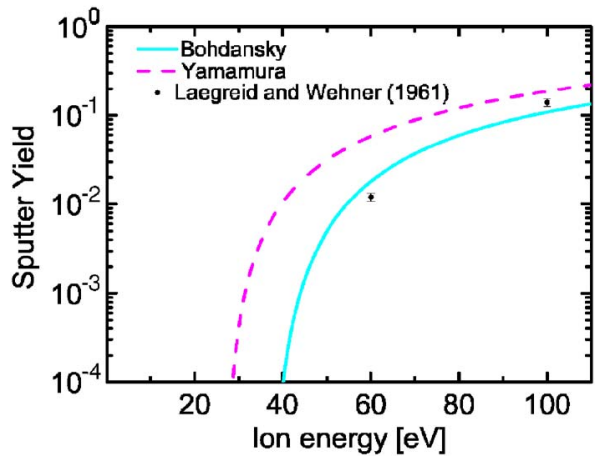

FIG. 8. (Color online) Sputter yield for $\mathrm{Ar}^{+}$on $\mathrm{Ru}$ as a function of ion energy according to the Bohdansky model (solid line), the Yamamura model (dashed line), and the experimental data of Laegreid and Wehner (circles).

above the sputter threshold. Therefore, we expect the damage to the mirror as a result of physical sputtering to be modest.

A word of caution is in place. Because the ion energies are close to the sputter threshold the calculated sputter rate is very sensitive to the value for the sputter threshold. For instance, the Yamamura [17] model for ion sputtering predicts a sputter threshold that is $10 \mathrm{eV}$ lower. Figure 8 shows the sputter yield according to the Bohdansky and Yamamura model together with the, to our knowledge, only available experimental data set [18] for this specific target-projectile combination.

If we convolute the sputter yield with the ion flux we obtain the total number of atoms removed per EUV pulse. For the Bohdansky and Yamamura model this amounts to, respectively, 6.2 and $205 \mathrm{~nm}$ per $10^{12}$ EUV pulses. Clearly, the Yamamura model predicts higher sputter rates than the Bohdansky model mainly due to the lower sputter threshold. The predicted sputter rates are too low to be experimentally verified. In the calculation of the sputter rate, we have only considered the impact of ions. However, charge-exchange collisions in the sheath region will result in a flux of fast neutrals to the mirror surface, that will also contribute to the sputter rate. In our low-pressure application, the neutral flux is very low, because the mean free path for charge-exchange collisions for energetic ions $(E=30-100 \mathrm{eV})$ is $\approx 10$ times higher than the thickness of the plasma sheath. Hence, sputtering by neutrals can be neglected.

\section{CONCLUSIONS}

In conclusion, we have demonstrated that with PICMCC simulations it is possible to describe transient plasmas in the nonlocal regime without the need for equilibrium assumptions for the fields or the velocity distributions of the charged particles. Furthermore, the EUV radiation driven plasma is found to be weakly ionized (ionization degree $<0.1 \%$ ) with a low plasma density $\left(n_{e}=4 \times 10^{15} \mathrm{~m}^{-3}\right)$.

The simulations show that the potential drop across the plasma sheath is dictated by the electron energy. Ions are accelerated across the sheath, but due to their inertia, most ions reach the mirror after the EUV pulse has ended.

Based on two models for the sputter yield, we conclude that only a small fraction of the ions impacting on the optics will have sufficient energy to sputter. However, the calculated sputter rate strongly depends on which model is applied, mainly because the two models employ a different value for the sputter threshold. Further experimental investigation is required to determine the value of the sputter threshold. The predicted sputter rate is very low $(<1 \mathrm{~nm}$ per $10^{9}$ EUV pulses) and cannot be measured directly. Future investigations should aim to verify the other predictions of the simulations regarding the ion impact energy distribution, plasma density, and electron energy distribution.

\section{ACKNOWLEDGMENT}

The authors are grateful to V. Ivanvov for the helpful discussions and suggestions.
[1] T. Ito and S. Okazaki, Nature (London) 406, 1027 (2000).

[2] E. R. Kieft, J. J. A. M. van der Mullen, G. M. W. Kroesen, and V. Banine, Phys. Rev. E 68, 056403 (2003).

[3] W. N. K. Bergmann, O. Rosier, and R. Lebert, Appl. Opt. 39, 3833 (2000).

[4] C. K. Birdsall and A. B. Langdon, Plasma Physics via Computer Simulation (Adam Hilger, London, 1991).

[5] R. W. Hockney and J. W. Eastwood, Computer Simulation Using Particles (Adam Hilger, London, 1988).

[6] V. Vahedi and M. Surendra, Comput. Phys. Commun. 87, 179 (1995).

[7] C. K. Birdsall and D. Fuss, J. Comput. Phys. 3, 494 (1969).

[8] A. V. Phelps and Z. L. Petrovic, Plasma Sources Sci. Technol. 8, R21 (1999).

[9] L. D. Landau and E. M. Lifshitz, Quantum Mechanics: Nonrelativistic Theory (Pergamon Press, Oxford, 1977).

[10] A. Okhrimovskyy, A. Bogaerts, and R. Gijbels, Phys. Rev. E
65, 037402 (2002).

[11] C. B. Opal, W. K. Peterson, and E. C. Beaty, J. Chem. Phys. 55, 4100 (1971).

[12] M. Surendra, D. B. Graves, and I. J. Morey, Appl. Phys. Lett. 56, 1022 (1990).

[13] S. Bajt, J. B. Alameda, T. W. B. Jr., W. M. Clift, J. A. Folta, B. Kaufmann, and E. A. Spiller, Opt. Eng. 41, 1797 (2002).

[14] C. W. Gwyn, R. Stulen, D. Sweeney, and D. Attwood, J. Vac. Sci. Technol. B 16, 3142 (1998).

[15] B. L. Henke, E. M. Gullikson, and J. C. Davis, At. Data Nucl. Data Tables 54, 181 (1993).

[16] J. Bohdansky, Nucl. Instrum. Methods Phys. Res. B 2, 587 (1984).

[17] Y. Yamamura and H. Tawara, At. Data Nucl. Data Tables 62, 149 (1996).

[18] N. Laegreid and G. Wehner, J. Appl. Phys. 32, 365 (1961). 